INPLASY

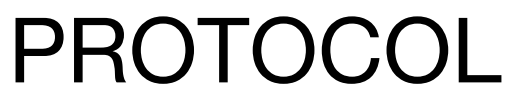

To cite: Fuentes-Aspe et al. Risk factors associated with the loss of physical functioning in critical care patients. A protocol of overview of systematic reviews and metaanalysis. Inplasy protocol 202090069. doi: 10.37766/inplasy2020.9.0069

Received: 16 September 2020

Published: 17 September 2020

Corresponding author:

Rocío Fuentes-Aspe

rocio.fuentes.aspe@gmail.com

Author Affiliation:

Doctorado en Metodología de la investigación biomédica y salud pública, Universidad Autónoma de Barcelona, España.

Support: None.

Review Stage at time of this submission: Preliminary searches.

Conflicts of interest:

No conflict of interest.

\section{Risk factors associated with the loss of physical functioning in critical care patients. A protocol of overview of systematic reviews and meta-analysis}

Fuentes-Aspe R1; González-Seguel, F2; Merino-Osorio, C3; Gutiérrez-Arias, R4; Marzuca-Nassr, GN5; Serón, P6.

Review question / Objective: What risk factors are associated with loss of physical functioning in critical care patients?

Condition being studied: Several studies that have described various risk factors or predisposing factors that would enhance or influence the patient to develop a greater degree of muscle weakness or further functional impairment. This leads to uncertainty as to whether the report or description of risk factors for this particular condition has covered or considered all studies in which the condition has been studied in all forms of designation. Hence, the purpose of this overview is to describe all reported or studied risk factors for developing or influencing loss of physical function in critically ill patients.

INPLASY registration number: This protocol was registered with the International Platform of Registered Systematic Review and Meta-Analysis Protocols (INPLASY) on 17 September 2020 and was last updated on 17 September 2020 (registration number INPLASY202090069).

\title{
INTRODUCTION
}

Review question / Objective: What risk factors are associated with loss of physical functioning in critical care patients?
Rationale: Muscle weakness and loss of function associated with human movement is one of the most characteristic findings in post-UCI survivors. The literature describes 
that between $25 \%$ and $60 \%$ of patients will develop some degree of skeletal muscle mass loss due to decreased contractile activity, bed rest and mechanical ventilation, conditioning skeletal and diaphragmatic muscle weakness. The concept of impairment of physical functioning of critically ill patients has been studied in different studies, describing a relationship between the deterioration of the systems (muscular represented by muscle strength), the limitation of activity (mobility), and the restriction of participation (level of independence in daily activities). Otherwise, there are several studies that have described various risk factors or predisposing factors that would enhance or influence the patient to develop a greater degree of muscle weakness, and therefore physical function, however, the heterogeneity of the concepts used in the various studies to refer to the same condition of interest is evident. This leads to uncertainty if the report or description of risk factors for this particular condition has covered or considered all studies in which the condition has been studied in all forms of designation. Having a synthesis and description of these factors or triggers is a relevant contribution to the prevention of loss of physical function after ICU. With the dissemination of the result of this Overview in the ICU medical community, it will be possible to establish strategies to reduce the incidence of acquired weakness in the ICU and subsequent post-ICU syndrome.

Condition being studied: Several studies that have described various risk factors or predisposing factors that would enhance or influence the patient to develop a greater degree of muscle weakness or further functional impairment. This leads to uncertainty as to whether the report or description of risk factors for this particular condition has covered or considered all studies in which the condition has been studied in all forms of designation. Hence, the purpose of this overview is to describe all reported or studied risk factors for developing or influencing loss of physical function in critically ill patients.

\section{METHODS}

Search strategy: The search will be made in electronic databases MEDLINE, EMBASE, Cochrane Library, CINAHL. The date of the search will be on september 2020. Terms with natural language and controlled vocabulary (MeSH or EMTREE, as appropriate) will be used. No year filters and without restriction of languages. The search strategy used is as follows: ("Critical Patient" OR "critically ill patient" OR "critical ill patient" OR "critically ill" OR "Critical Care"[Mesh] OR "Critical IIIness"[Mesh] OR "Intensive Care Units"[Mesh] OR "Critically ill" OR "Critical Illnesses" OR ICU OR "critical care unit") AND ("Intensive care unit-acquired weakness" OR ICUAW OR "ICU-acquired weakness" OR "Sarcopenia"[Mesh] OR "Muscular Atrophy"[Mesh] OR "Muscle Weakness"[Mesh] OR "Atrophy"[Mesh] OR "muscle weakness" OR "loss of muscle mass" OR "muscle atrophy" OR "muscle degeneration" OR Frailty OR "muscle wasting"OR "physical frailty" OR frailty OR "Physically frail" OR "physical functioning" OR "physical function" OR "physical strength" OR "functional capacity" OR "physical activity levels") AND ("Risk Factors"[Mesh] OR Trigger OR "risk factors" OR "Precipitating Factors"[Mesh] OR "Risk"[Mesh] OR triggering).

Participant or population: The population under study is critical patients, adults, over 18 years old, hospitalized in critical patient units.

Intervention: In this overview will be considered systematic reviews that have analyzed the risk factors in the generation of a deterioration in physical function or any condition that is within the domains defined by the ICF "World Health Organization's International Classification of Functioning" to assess the physical functioning in critically ill patients.

Comparator: Exposure not will be compared.

Study designs to be included: This overview will consider systematic reviews 
with or without meta analysis. The primary studies included in the selected systematic reviews should consider observational studies, prospective cohort studies.

Eligibility criteria: This overview will consider systematic reviews that include studies with participants admitted and hospitalized in the intensive care unit (ICU), over 18 years old.

Information sources: The search will be made in electronic databases MEDLINE, EMBASE, Cochrane Library, CINAHL. The date of the search will be on september 2020. Terms with natural language and controlled vocabulary (MeSH or EMTREE, as appropriate) will be used.

Main outcome(s): The main result of this overview will be the associated risk factors reported for loss of physical functioning (or any other concept that describes this condition) in intensive care patients. Selected systematic reviews should include primary studies that analyze the outcome of interest in this overview. The included reviews may or may not have performed a meta-analysis, if so, the result would be the relative risk or the odd ratio to develop or favor for loss of physical functioning.

Additional outcome(s): Additional results related to the loss of physical functioning condition in ICU patients, such as mortality, adverse events, functional capacity, quality of life, will be reported.

Data management: Once the search is complete, all the identified elements will be incorporated into the COVIDENCE $®$ software for selection with the criteria described and elimination of duplicates. Titles and abstracts will be reviewed by two independent, blinded researchers. Potentially relevant studies will be reviewed in their full text for selection or final exclusion. The reasons for excluding fulltext studies that do not meet the inclusion criteria will be recorded and reported in the review. Any disagreement that arises between the reviewers at each stage of the study selection process will be resolved by discussion or by a third reviewer. Two independent reviewers will obtain data from the articles included using a data extraction table developed by the authors, any disagreement that arises between the reviewers will be resolved through discussion, or by a third reviewer. The data to be extracted are: 1. Characteristics of the systematic reviews included: author, year and journal of publication, number of primary studies included, inclusion criteria for the selection of primary studies (Participants/population, types of study included), databases consulted, main outcome(s) of the review, risk of bias's tool, and method of statistical analysis if it used. 2. Primary outcome; risk factors reported for loss of physical functioning (or any other concept that describes this condition), and the result of the risk analysis of developing the event of interest. 3. Secondary outcome; instruments used to measure the variables and the result.

Quality assessment / Risk of bias analysis: The assessment of the risk of bias of the included studies will be carried out with the ROBIS tool, designed for the critical analysis of systematic reviews or metaanalyses but specifically for the development of a systematic review. The risks of bias are analyzed in relation to the particular design, conduct, and synthesis of a systematic review.

Strategy of data synthesis: The results of the search will be fully reported in the final document and presented in a proposed flowchart for exploratory type reviews proposed by "Preferred Reporting Items for Systematic Reviews and Metaanalyzes" (PRISMA). The strategy for data synthesis will consist first of obtaining the qualitative data from each review, which will be entered into the same screening software as the studies (COVIDENCE®) and in a data extraction table. The results will be reported descriptively in the text, and in tables. The results of selected and excluded studies will be reported. A synthesis of the characteristics of the included studies, and the result and description of the risk factors found, will be described. The Tables will include: Table N1 will include the characteristics of the 
selected studies, Table 2 the result of the risk of bias applied to the included reviews. Table 3 the synthesis of the risk factors and their results found. And a 4 Table with the instruments used to assess physical functioning.

Subgroup analysis: Due to the diversity of pathologies for which patients can be hospitalized in an intensive care unit, it will be appropriate to describe by subgroups or by subtypes of pathologies the findings that are reported. For example, it will be considered to present the results by types of pathologies: of neurocritical, cardiovascular, respiratory, surgical, traumatic origin, others, and the risk factors studied in each group by pathology and the results.

Sensibility analysis: It is not within the objectives to re-meta-analyze, therefore a sensibility analysis will not be performed.

Language: Without restriction of languages.

Country(ies) involved: Chile.

Keywords: "Critical illness", "Physical function", "Intensive care unit-acquired weakness", "risk factors", "post-UCI survivors".

Dissemination plans: Once the overview is completed, it will be edited in the format of a medical scientific journal, to which it will be submitted for publication. The focus of the journal will be on journals with an interest in critical care, physical therapy or rehabilitation.

Contributions of each author:

Author 1 - Rocío Fuentes-Aspe - lead author, developed general idea, wrote protocol and manuscript.

Author 2 - Felipe González-Seguel - coauthor, participates in the development of the general idea, reviews protocol and manuscript.

Author 3 - Catalina Merino-Osorio - coauthor, participates in the development of the general idea, reviews protocol and manuscript.
Author 4 - Ruvistay Gutiérrez-Arias - coauthor, participates in the development of the general idea, concepts, reviews protocol and manuscript.

Author 5 - Gabriel Nasri Marzuca-Nassr co-author, participates in the development of the general idea, concepts, reviews protocol and manuscript.

Author 6 - Pamela Serón - co-author, participates in the development of general idea, methodology, reviews protocol and manuscript.

Organisational affiliation of the systematic review: Departamento de Medicina Interna, Universidad de La Frontera, Temuco, Chile. 